\title{
A novel method for localising a randomly distributed wireless sensor network
}

Ahmad A. A. Alkhatib, 1

Mohammad Alia, 1

Adnan Hnaif, 1

Sufian Yousef, 2匹

Email sufian.yousef@anglia.ac.uk

1 Al Zaytoonah University of Jordan, Amman, Jordan

2 Anglia Ruskin University, Cambridge, UK

Received: 12 June 2017

\section{Abstract}

Wireless sensor networks are dependent on sending and receiving signals; the system will not be capable of functioning if communication between sensors is not established. Localisation is one of the most important functions in this technology to localise nodes, events or the data source. In this study, we present a new method for outdoor randomly distributed nodes with no need for any excess devices, such as GPS devices or directional antennas or ultrasonic sensors. The method is based on using only the simple node component to provide the node and event position and has the ability to adapt mobility and scalability without affecting network functionality. All of the results are based on an ideal environment. AQ1

Keywords 
Wireless sensor network (WSN) localisation

WSN outdoor localisation

Efficient and cheap method for sensor localization

\section{Introduction}

One of the main functions in wireless sensor networks is selflocalising, where sensors are supposed to be capable of localising themselves by using different techniques. Most localisation methods use estimation algorithms, which utilise directional antennas and a measurement, such as angle of arrival (AOA), time of arrival (TOA), time difference of arrival (TDOA) or received signal strength (RSS), to apply them in equations to localise themselves (Rong and Sichitiu 2006; Kulakowski et al. 2010). A different method involves adding GPS devices to the sensors. Sometimes a GPS device is added to only a few of the sensors and all of the others localise themselves terrestrially (de Oliveira et al. 2007; Conrad et al. 2009). The final option is to add ultrasonic sensors at each node to perform the localisation (libelium 2013).

In wireless sensor networks, cost and size are important. Adding devices, such as GPS devices and directional antennas, or even an array of antennas for localisation purposes, will increase the cost, size and power consumption of the sensor device. Directional antennas will limit broadcasting in some applications, such as fire detection. Use of GPS leads to more power consumption. Estimation algorithms require extensive complicated calculations. Use of the estimation algorithms will be problematic for sensors with regard to limited resources, limited memory and a limited buffer in addition to the inaccuracy and NLOS (Conrad et al. 2009).

\section{Related work}

In general, the wireless sensor network localisation algorithm uses either GPS devices (Jurdak et al. 2013) or estimation algorithms that use directional antennas with a measurement, such as AOA (Jiang et al. 2012; Luo et al. 2013), TOA (Yu et al. 2009; Mekonnen and Wittneben 2014; Go and Chong 2015), TDOA (Yang and Ho 2009; Cui et al. 2013; Cheng et al. 2012) or RSS (Sahu et al. 2013; Jun 2014; Mukhopadhyay et 
al. 2015), to apply the measurements in equations to localise the sensors.

The estimation techniques can be divided into the following (Niculescu and Nath 2003, Rao 2005, Boudhir et al. 2010, Kulakowski et al. 2010):

- Angle of arrival measurement: AOA technique based on the amplitude responses and phase of reception on antennas, where angle of reception determined by the signal strength and beam form. Due to amplitude and phase variation it is almost impossible to define the received signal angle. However, this problem solved by adding omnidirectional antenna with a static beam form, the phase response can be defined by comparing the two beam forms. Another method is to use two or four directional antennas to perform an overlapping between beam forms.

- Distance related measurements: this technique can measure:

(a) One-way propagation time: device precise synchronisation to measure time propagation then calculate the distance.

(b) Round trip propagation: calculate the distance by measure the round trip propagation time. Signal processing in the second node will cause some errors.

(c) Time difference of arrival (TDOA): coordination estimation of the transmitter based on measuring the signal on multiple receivers. Then using measurement in some calculations such as; cross-correlation.

(d) Received signal strength (RSS): using the signal strength at the receiver to use it in the appropriate model of the propagation models to calculate the distance. This technique define only distance, not directions.

\section{AQ2}

Cheng et al. (2012) reviewed the available localisation techniques. They divided the techniques into target localisation and node selflocalisation. The following figure shows the localisation method taxonomy that they suggested (Fig. 1). 
Fig. 1

Localisation method taxonomy (Cheng et al. 2012)

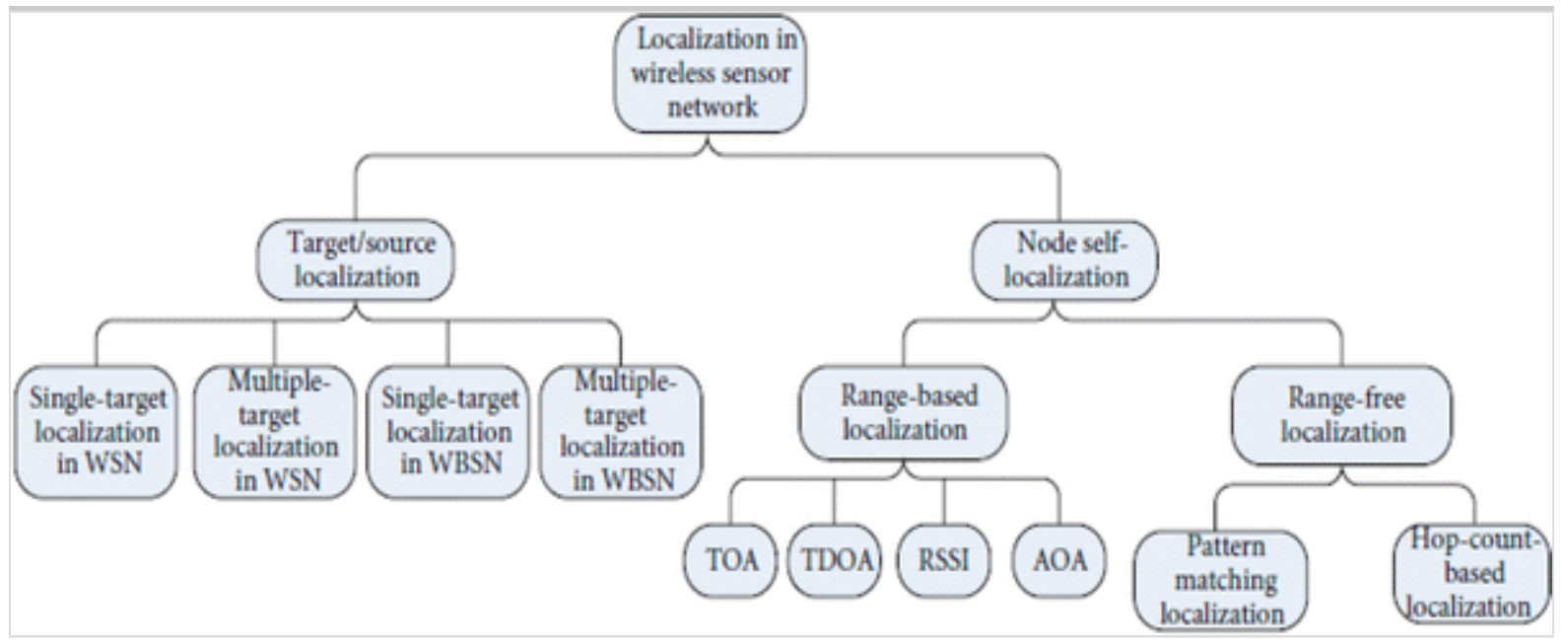

AQ3

Furthermore, the authors covered special scenarios, such as non-line of sight (NLOS), where they explained the parametric and nonparametric solutions. LOS and NLOS problems are major causes of errors in localisation measurement methods. The localisation measurement methods have been the focus of many researchers, such as Li et al. (2015), who proposed the Gaussian mixed model-based nonmetric multidimensional (GMDS) to solve these problems. The main contribution in the proposed GMDS solution is estimating RSSI using a Gaussian mixed model (GMM) and then manipulating the results in a multidimensional scaling (MDS) matrix and comparing this matrix with relative anchor coordinates.

Kuriakose et al. (2013) divided the localisation techniques into five types based on the available methods:

- Known location, using GPS;

- Proximity based, using IR or Bluetooth;

- Angle based, using AOA;

- Range based, using RSSI, TDOA and TOA; and

- Distance based, DV Hop or DV Distance. 
The conclude their paper with a comparison table that shows the localisation techniques and their accuracy, as in the following Table 1.

\section{Table 1}

Comparison of accuracy among localisation techniques (Kuriakose et al. 2013)

\section{Localisation techniques used}

GPS

Angle-based approach

Range-based approach

DV-based approach
Accuracy (m)

$2-15$

$1-6$

$4-8$

$10-20$

\section{AQ4}

Alrajeh et al. (2013) stated that localisation schemes are classified as anchor-based or anchor-free, centralised or distributed, GPS-based or GPS-free, fine grained or coarse grained, stationary or mobile sensor nodes, and range-based or range-free.

They clarify the five location estimation concepts as follows.

- Lateration: estimate location by using measuring distance.

- Angulation: estimate location by measuring angles.

- Trilateration: estimate location by measuring the distance from three reference nodes and then consider these distances as the half diameter of the three imaginary circles created between the node and the reference nodes. Then, the intersection is the required location.

- Multilateration: more than three references are used to estimate locations.

- Triangulation: measure the angles with two reference nodes to estimate the location by using trigonometric laws. 
$\mathrm{Xu}$ et al. (2013) focused on the localisation of an unmanned aerial vehicle (UAV), in which the arial beacon assisted localisation (ABAL)the proposed solution-used the RSSI as well as the number of beacon nodes to provide the location. The method used RSSI for triangulation and four virtual beacons to reduce the errors in the NLOS problems.

Mistry and Mistry (2015) focused on the use of RSSI in the localisation process to reduce errors where Adewumi et al. (2013) used crossbow IRIS to test indoor distance estimation in sensor network. The model evaluation and analysis contribute to more accurate results. Shojaifar (2015) proposed a method for localising moving targets using RSSI. He conducted a 30 experiment to show that: (1) the results pointed that the direct path between nodes can improve accuracy. (2) The target node's antenna type can effect on the RSSI values and distance measurement error.

Based on this study, all previous studies use one or more of the six methods: GPS, ultrasonic sensors, AOA, TOA, TDOA and RSSI. The localisation service is one of the main contributions from this research, where a new method is developed and tested. More details are provided in the following sections.

\section{The state of the art}

This work presents a new localising method for wireless sensor networks that simplifies the network requirement for extra components, antennas and calculations to provide this service through the network. The mathematical model and network installation show the simplicity and efficiency that makes this method suitable for many applications. This method considers the ideal environment in the calculations.

This work differs from other studies in the following points:

- No need to use GPS devices or ultrasonic sensors.

- No need to use directional antennas and measure the AOA, TOA, and TDOA. We only need an omnidirectional antenna.

- No need to install large numbers of anchor nodes to ensure that 
the nodes lie between three different anchors to perform the normal method of triangulation.

- Scalability and mobility are problems in other techniques, especially a normal triangulation technique, where mobility and scalability are problems. These problems are solved in the proposed localisation method. The network coverage can be extended anywhere, and new nodes can join the network any time without affecting network functionality. Node mobility issues are solved, as well.

\section{Proposed localisation method}

A simple localisation method is based on fixing some nodes as reference nodes, and all of the other nodes can then localise themselves by measuring the power received for localisation messages coming from neighbours. The measured power received is used in some mathematical triangulation calculations to find the node position relative to the sink and neighbours. The triangulation in this method is different from the triangulation used in ad hoc, mobile or other systems. Normally, triangulation is based on spreading a number of reference points throughout the coverage area to ensure that any device located between three reference base stations performs the triangulation (Fidis 2013; Guoqiang et al. 2007). However, this localisation method only requires a minimum of three reference nodes that fixed somewhere around the sink. There is no need to spread reference nodes throughout the coverage area or to use powerful devices, such as anchors or nodes with transonic sensors or others, for reference nodes.

The localisation algorithm can be performed by carrying out a transmission and reception and then a calculation and logical investigation. A group of conditions must be applied to the results to find the right location for the required node. The following is a flow chart that explains the entire localisation method (Fig. 2).

\section{Fig. 2}

Triangulation in other systems 


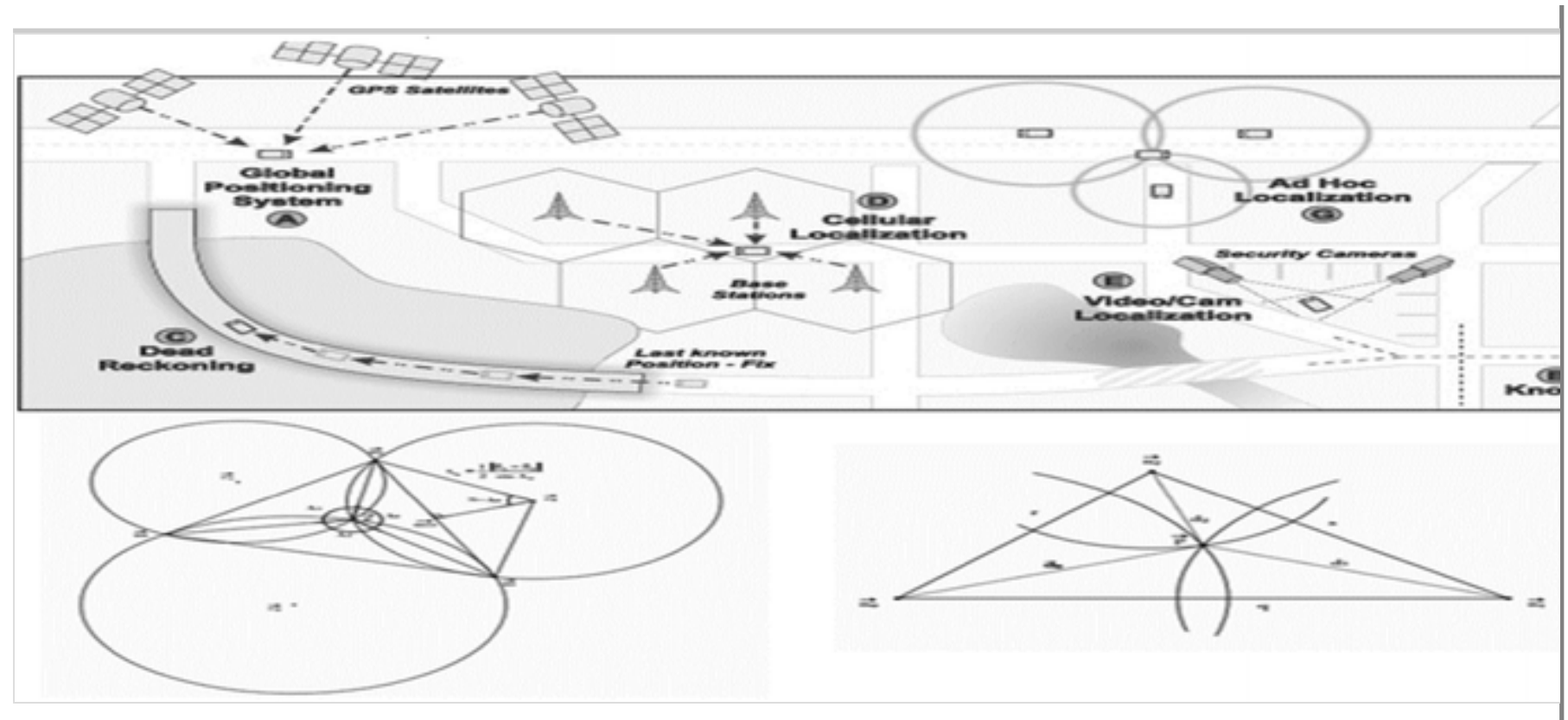

(a) Advantages of the assumed system.

As described before, the network must be set up first, and the reference nodes must be fixed around the sink. Then, we can spread the sensors randomly, with reasonable distances between them (depending on the application). The localisation method can be performed by following the steps below.

- All of the reference nodes must broadcast a localisation message that carries the location information of the reference node itself.

- Unknown sensors will receive the messages from the reference node. At least three messages or the sensor must wait until more reference nodes become available.

- By applying the measured/received signal strength RSSI in the appropriate propagation models, each node can calculate the distances between the node itself and the senders (reference nodes) (Rao 2005).

\section{The network setup}

This research is based on a certain pre-installation:

- Sensors are distributed randomly in the coverage area, where reasonable distances must be considered. The distances depend on 
the range of the sensors used; some of them can sense the environment over $1 \mathrm{~m}$ and others can sense the environment up to $45 \mathrm{~km}$, such as FireWatch, the German System (FireWatch 2013; Alkhatib 2014).

- The sink can be installed anywhere within the network range, depending on the environment. E.g., in forests, the location is preferred at the edge-close to the urban area to make it easier for an external network connection, e.g., mobile network, satellite network, microwave or Internet. In this research, the sink is assumed to be in the middle of the network range, but the method is designed to adapt to any position for the sink.

- The sink must be fixed and provided with its own position information as a starting point.

- The sink is always the reference point.

- Several reference nodes, a minimum of three nodes, must be installed at a fixed known position around the sink with a reasonable distance and angles between them. In this research, several nodes were used as reference nodes installed around the sink (Fig. 3).

\section{Fig. 3}

WSN network setup. a Setting references, b scattering nodes randomly

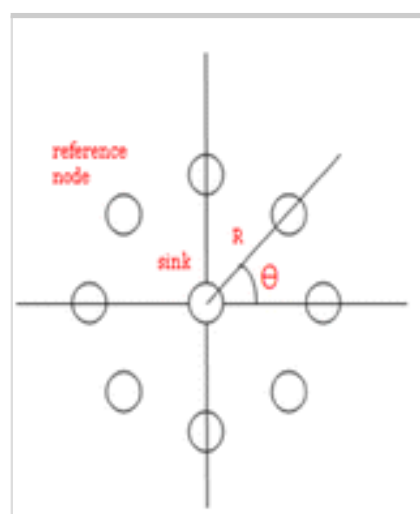

(a)

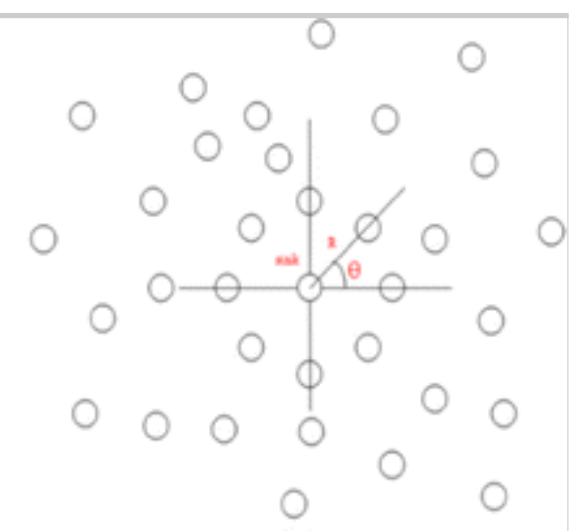

(b) 


\section{The rest of the technique summarized in the following table:}

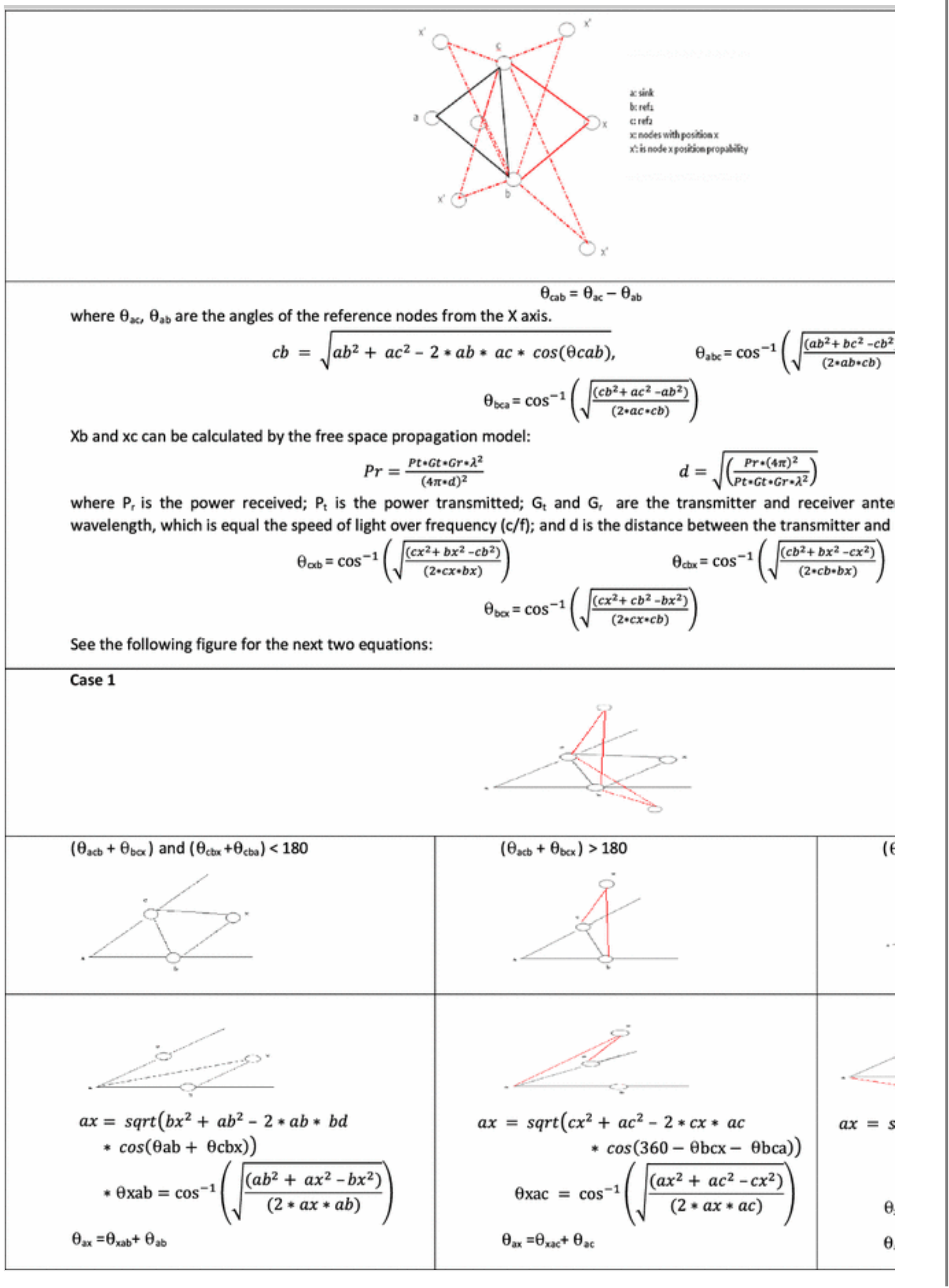




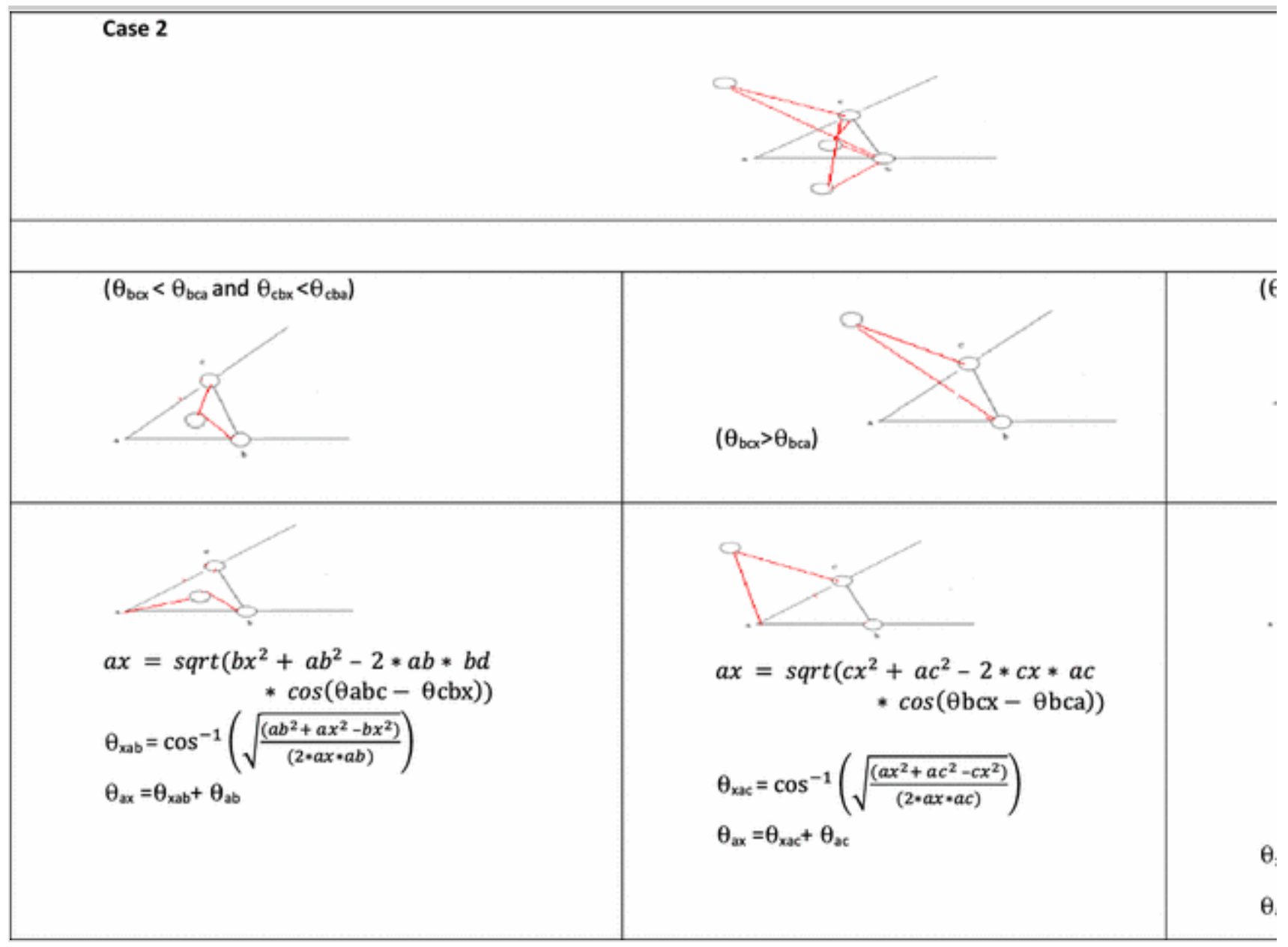

- Finally, all nodes that know their own location should send a localisation message to help other nodes to localise themselves. This time, the new nodes can be considered to be reference nodes to spread the localisation over a wider range. This process will keep going until all of the nodes have their own location information.

This method can adapt to mobility, scalability and node failure.

- Mobility: mobility can be performed by checking the neighbours regularly. If the neighbour list has any changes for any node, localisation must be performed for this node again and the sink and neighbour list must be updated. Localisation can be performed only for this node without affecting the network functionality.

- Scalability: if any new nodes joined the network for any reason, 
such as adding a new coverage area to the network, replacing dead battery nodes or burnt nodes with new nodes or filling coverage gaps that occur for any reason, they can receive localisation messages from neighbours and go through the method without affecting the functionality of other nodes or the network in general. See the flowchart below.

- Node failure: because we can localise all of the sensors, we can use the node location information in a graphical user interface (GUI) for operators. By this, we can present any updates on the network members. If any node loses its connection with the network or is not located anywhere else in the network, it will be considered a node failure and can be presented in the GUI so that the operator can indicate whether there is a coverage gap in the network. This property is assessed through the coverage method and is presented in MATLAB. See the coverage section (Fig. 4).

Fig. 4

Scalability flow chart

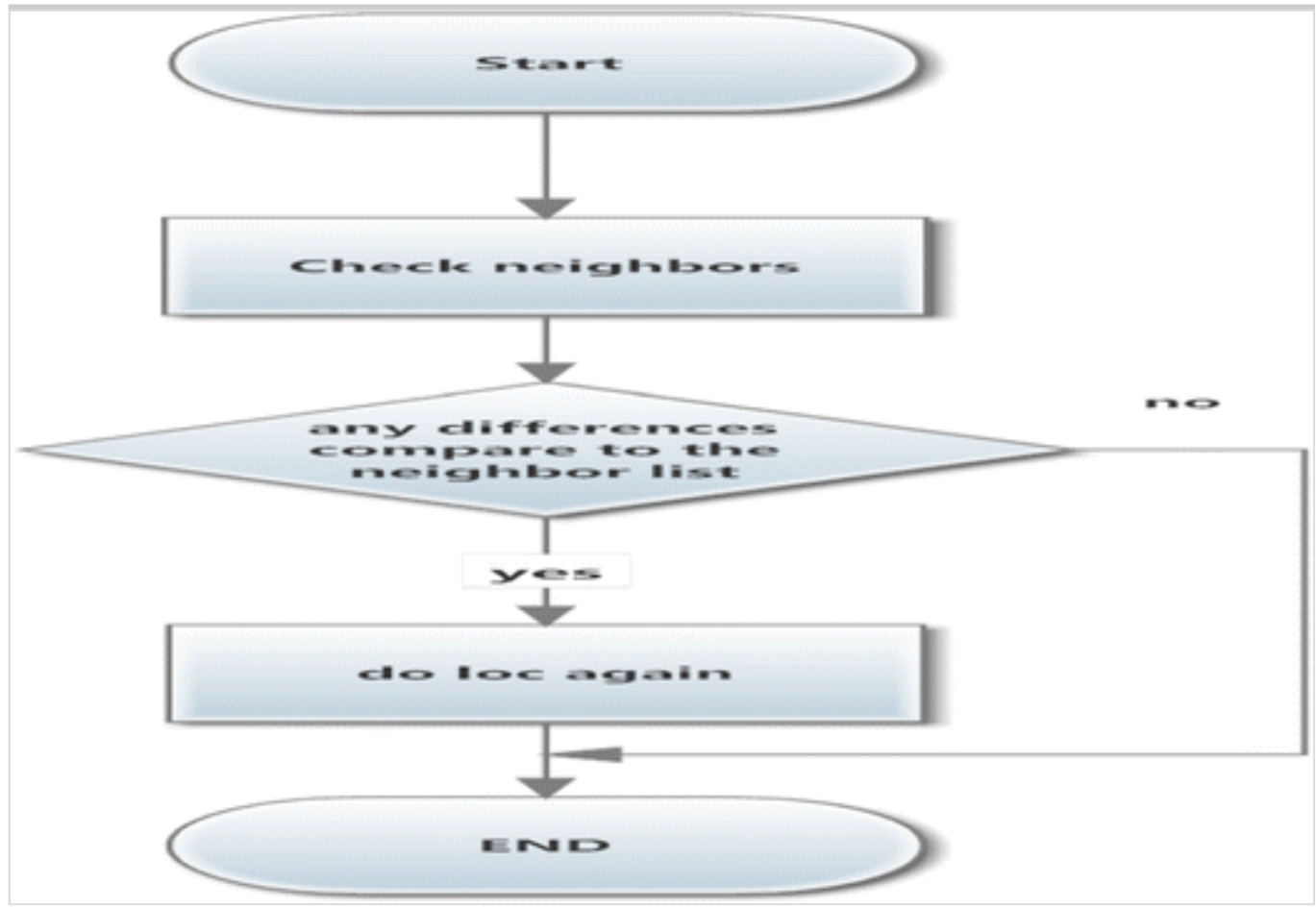

6. Result and analysis 
To verify and validate the localisation method, 50 nodes are tested in NS2, in which 15 are used as reference points and 35 nodes are used randomly. The obtained results were very good. The method was tested with error model (Figs. 5, 6).

$$
\mathrm{R} \text { Error } \%=\sum_{n=15}^{49} \frac{\mathrm{ABS} \mid \mathrm{R} \text { simulation }-\mathrm{R} \text { calculation } \mid}{\mathrm{R} \text { calculation }} \times 100 \%
$$

$\mathrm{R}$ Error $\%=\frac{3.023144}{1317.26} \times 100 \%=0.002295 \times 100 \%=0.2295 \%$

$$
\begin{aligned}
& \theta^{\circ} \text { Error } \%=\sum_{n=15}^{49} \frac{\operatorname{ABS} \mid \theta^{\circ} \text { simulation }-\theta^{\circ} \text { calculation } \mid}{\theta^{\circ} \text { calculation }} \times 100 \% \\
& \theta^{\circ} \text { Error } \%=\frac{3.951734}{5682.847} \times 100 \%=0.000695 \times 100 \%=0.0695 \%
\end{aligned}
$$

the error percentages in $R \& \theta^{\circ}$ are very small. As a point of interest, when distributing the reference nodes randomly, the error percentage is reduced in $\mathrm{R} \& \theta^{\circ}$.

\section{Fig. 5}

wpan.scn

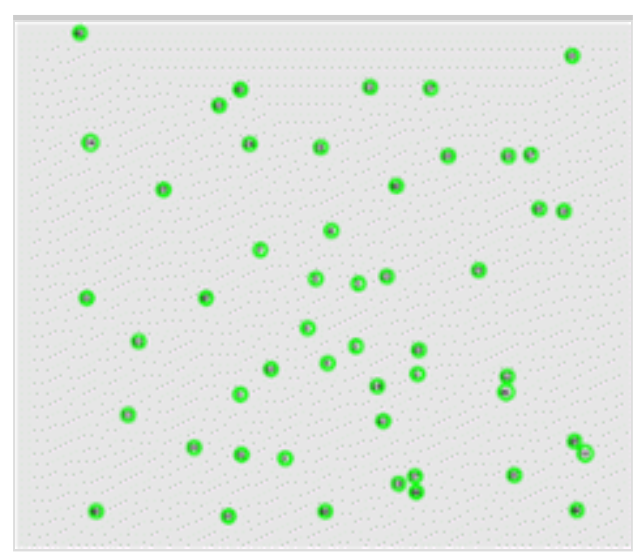

Fig. 6 
$\mathrm{R} \& \theta$ calculation and $\mathrm{R} \& \theta$ simulation

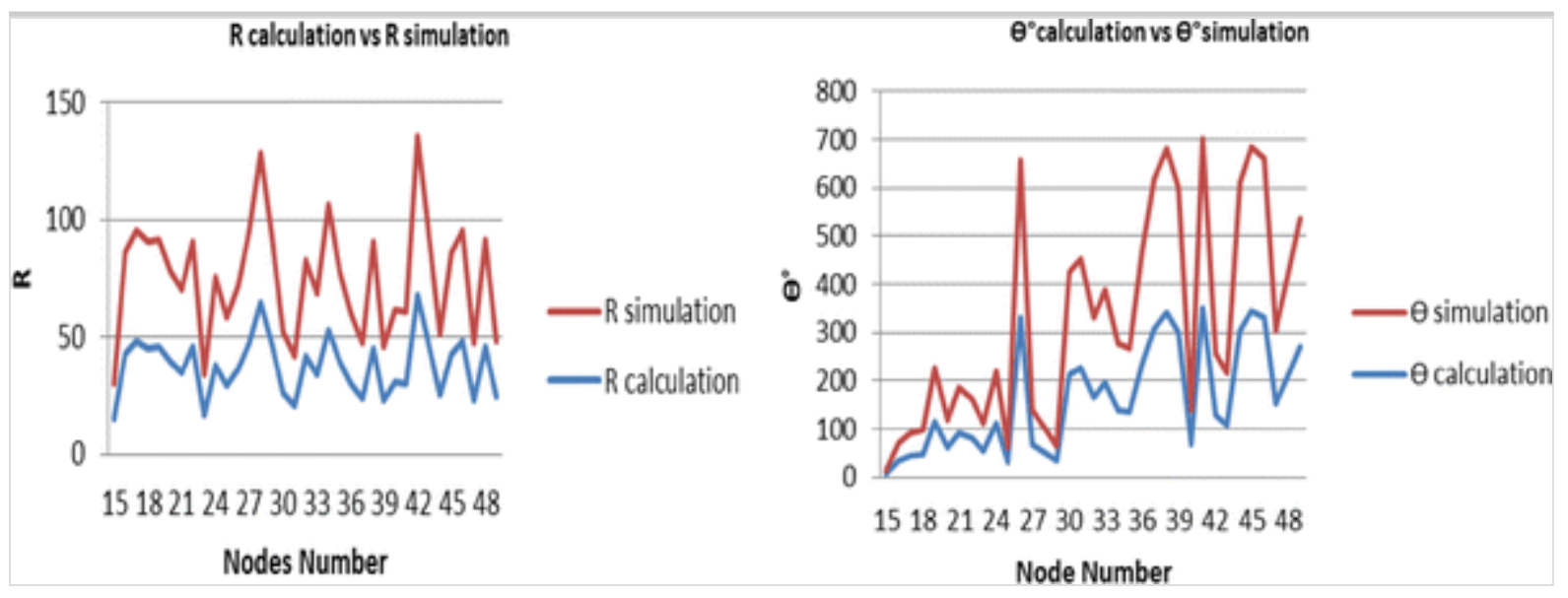

AQ6

\section{Conclusion}

Localization in sensor network is a very important issue, it does add extra cost on the network to localize nodes and increase nodes power consumption, since it mostly devices added to nodes. The proposed technique is only based on the basic component of the nodes to make each node able of localize itself, by setting the network with a start point (sink) and few reference nodes then start the localization calculation.

\section{References}

Adewumi O, Djouani K, Kurien A (2013) Performance evaluation of RSSI based distance measurement for localization in wireless sensor networks, vol 119. Springer, Berlin

Alkhatib AA (2014) A review on forest fire detection techniques. Int J Distrib Sens Netw 10

\section{AQ7}

Alrajeh N, Bashir M, Shams B (2013) Localization techniques in wireless sensor networks. Int J Distrib Sens Netw 9:304628 
Boudhir A, Mohamed B, Mohamed BA (2010) New technoique of wireless sensor networks localization based on energy. Int J Comput Appl 9:25-28

AQ8

Cheng L, Wu C, Zhang Y, Wu H, Li M, Maple C (2012) A survey of localization in wireless sensor network. Int J Distrib Sens Netw 8(12):962523

Conrad A, Liu Q, Russell J, Lalla J (2009) Enhanced forest fire detection system with GPS Pennsylvania

AQ9

Cui W, Wu W, Wang Y, Shan Y (2013) A gossip-based TDOA distributed localization algorithm for wireless sensor networks. In: 2013 2nd international symposium instrumentation and measurement, sensor network and automation (IMSNA), pp 783-788, $23-24$

de Oliveira H, Nakamura E, Loureiro A, Boukerche A (2007) Localization in time and space for sensor networks. In: 21st international conference on advanced information networking and applications, 2007 (AINA'07)

Fidis. Mobility and LBS. EU's 6th Framework Programme. Retrieved 11/4, 2013, from

http://www.fidis.net/resources/deliverables/mobility-andidentity/int-d1110001/doc/2/single/

FireWatch (2013) An early warning system for forest fires, successfully in the global use. http://www.firewatch.de/systemoverview

Go S, Chong J (2015) Improved TOA-based localization method with BS selection scheme for wireless sensor networks. J ETRI 37(4):707716

Guoqiang M, Barış F, Brian DO (2007) Wireless sensor network localization techniques. J Comput Netw 51(10):2529-2553 
Jiang J, Lin C, Lin F, Huang F (2012) ALRD: AoA localization with RSSI differences of directional antennas for wireless sensor networks. In: 2012 international conference on information society (i-Society), pp 304-309

Jun L (2014) Range error correction in RSSI-based wireless sensor node localization. In: 2014 IEEE international conference on mechatronics and automation (ICMA), pp 379-383

Jurdak R, Corke P, Cotillon A, Dharman D, Crossman C, Salagnac C (2013) Energy-efficient localization: GPS duty cycling with radio ranging. ACM Trans Sens Netw 9(2), Article 23

Kulakowski P, Vales-Alonso J, Egea-Lopez E, Ludwin W, García-Haro J (2010) Angle-of-arrival localization based on antenna arrays for wireless sensor networks. Comput Electr Eng 6:1181-1186

Kumar P, Reddy L, Varma S (2009) Distance measurement and error estimation scheme for RSSI based localization in wireless sensor networks. In: 2009 fifth IEEE conference on wireless communication and sensor networks (WCSN)

AQ10

Kuriakose J, Joshi S, George VI (2013) Localization in wireless sensor networks: a survey. In: Control instrumentation system conference (CISCON'2013)

Li B, Cui W, Wang B (2015) A robust wireless sensor network localisation algorithm in mixed LOS/NLOS scenarios. Sens J $15: 23536-23553$

libelium (2013) Ultrasound sensor on 3V3 Socket Reading. Retrieved 11/4, 2013, from http://www.libelium.com/development/waspmote/examples/sm-7ultrasound-sensor-on-3v3-socket-reading/

Luo J, Zhang X, Wang Z (2013) A new passive source localization method using AOA-GROA-TDOA in wireless sensor array networks and its Cramér-Rao bound analysis. In: 2013 IEEE international 
conference on acoustics, speech and signal processing (ICASSP), pp 4031-4035

Mekonnen ZW, Wittneben A (2014) Robust TOA based localization for wireless sensor networks with anchor position uncertainties. In: 2014 IEEE 25th annual international symposium personal, indoor, and mobile radio communication (PIMRC), pp 2029-2033

Mistry HP, Mistry NH (2015) RSSI based localization scheme in wireless sensor networks: a survey. In: Proceedings of the 2015 fifth international conference on advanced computing and communication technologies (ACCT'15). IEEE Computer Society, Washington, DC, PP 647-652. doi:10.1109/ACCT.2015.105

Mukhopadhyay B, Sarangi S, Kar S (2015) Performance evaluation of localization techniques in wireless sensor networks using RSSI and LQI. In: 2015 twenty first national conference communications (NCC), pp 1-6

Niculescu D, Nath B (2003) Ad hoc positioning system (APS) using AOA. In: IEEE INFOCOM

Rao V (2005) The simulative investegation of ZibBee/IEEE 802.15.4. Department of Electrical Engineering And Information Technology. Dresden University of Technology. Master of Science

Rong P, Sichitiu M (2006) Angle of arrival localization for wireless sensor networks. In: 3rd annual IEEE communications society on sensor and ad hoc communications and networks, 2006 (SECON'06)

Sahu PK, Wu EH-K, Sahoo J (2013) DuRT: dual RSSI trend based localization for wireless sensor networks. Sens J IEEE 13(8):31153123

Shojaifar A (2015) Evaluation and improvement of the RSSI based localization algorithm received signal strength indication (RSSI). Master of Science in Software Engineering, Faculty of Computing Blekinge, Institute of Technology 
Xu Y, Shi X (2013) ABAL: arial beacon assisted localisation of wireless sensor networks with RSS maxima. J Ad Hoc Sens Wirel Netw 29:93-112

Yang L, Ho KC (2009) An approximately efficient TDOA localization algorithm in closed-form for locating multiple disjoint sources with erroneous sensor positions. IEEE Trans Signal Process 57(12):45984615

Yu K, Guo YJ, Hedley M (2009) TOA-based distributed localisation with unknown internal delays and clock frequency offsets in wireless sensor networks. J Signal Process IET 3(2):106-118 